\title{
Le JCR facteur d'impact (IF) et le SCImago Journal Rank Indicator (SJR) des revues françaises : une étude comparative
}

\author{
Joachim Schöpfel \\ Hélène Prost
}

\section{(1) Introduction}

Une des fonctions principales des revues scientifiques est de contribuer à l'évaluation de la recherche et des chercheurs. Le débat actuel autour du nouveau dispositif de la recherche en France (ANR ${ }^{1}, \mathrm{AERES}^{2}$, autonomie des universités, réforme du CNRS etc.) a souligné cette fonction. L'accent est mis sur le mode de calcul d'indicateurs à partir du nombre de citations d'une publication scientifique. ${ }^{3}$

Depuis les premiers travaux d'Eugene Garfield il y a plus de 50 ans, le facteur d'impact (impact factor ou IF) répertorié dans le Journal Citation Reports $(J C R)^{4}$ de l'Institute for Scientific Information (Thomson Scientific) est devenu l'indicateur dominant de la qualité d'une revue (Garfield 1955, 1972 et 2006, Magri et al. 1996). L'attribution d'un facteur d'impact augmente la valeur marchande d'une revue. Publier dans une revue avec IF contribue au statut d'un chercheur et au rayonnement de son organisme de recherche.

Or, le nombre d'études critiques sur le facteur d'impact ne cesse de grandir. Les reproches sont précis $^{5}$ :

- Comparer les revues de différents domaines est plus ou moins impossible.

- La part de revues avec IF dans les domaines scientifiques est inégale (problème de représentativité).

- Les autocitations (tout comme d'autres stratégies de citation éditoriales) induisent un biais non corrigé.

- La qualité d'une citation n'est pas prise en compte.

- Le prix du Web of Science est trop élevé.

- Le nombre de revues scientifiques est limité, notamment pour des revues récentes.

- Les revues anglophones sont surreprésentées.

Les chercheurs français ont surtout critiqué ce dernier aspect (cf. par exemple Ojasoo et al. 2002). L'absence d'indexation par l'ISI de la plupart des titres français est un handicap aussi bien pour les chercheurs français que pour les éditeurs francophones ; publier en français est de ce fait défavorisé.

Une équipe de recherche espagnole a développé en 2007 un indicateur (SCImago Journal Rank Indicator ou indicateur SJR) ${ }^{6}$ qui applique l'algorithme de Google (PageRank) aux

\footnotetext{
${ }^{1}$ ANR : Agence Nationale de la Recherche. Cf. http://www.agence-nationale-recherche.fr/

${ }^{2}$ AERES : Agence d'Evaluation de la Recherche et de l'Enseignement Supérieur. Cf : http://www.aeresevaluation.fr/

${ }^{3}$ Pour une introduction générale, cf. Durand-Barthez 2006.

${ }^{4}$ Le $J C R$ est commercialisé via le produit Web of Science (WoS). En fait, il s'agit de deux éditions, l'un pour les STM, l'autre pour les SHS.

${ }^{5}$ Cf. par exemple Pinhas \& Kordon 1997, Pocard 1998, Baudoin et al. 2004, Dong et al. 2005
} 
revues indexées par la base SCOPUS d'Elsevier. Cette solution présente plusieurs avantages par rapport au IF de Thomson Scientific :

- Les autocitations d'une revue sont exclues.

- La qualité d'une citation est prise en compte par le biais de l'algorithme PageRank, c'est-à-dire chaque citation est pondérée en fonction du prestige de la revue citant (cf. Page et al. 1998 et Bollen et al. 2006).

- Le produit SCImago est librement accessible sur le Web.

- La couverture de la base $S C O P U S$ est plus large que celle du $J C R$ et tend à réduire la surreprésentation des titres de langue anglaise (cf. de Moya et al. 2007, Gavel \& Iselid 2008 et Falagas et al. 2008b).

L'intérêt de SCOPUS pour des analyses bibliométriques a été confirmé plusieurs fois (cf. Ball \& Tunger 2006, Neuhaus \& Daniel 2008). Ainsi, le SJR devient un concurrent direct de l'IF.

Falagas et al. (2008a) viennent de publier une première comparaison directe des deux indicateurs IF et SJR. Les auteurs détaillent les caractéristiques des deux indicateurs et comparent le résultat de leur mode de calcul, notamment par rapport au rang des revues. Le résultat est encourageant pour le SCImago Journal Rank Indicator qu'ils considèrent comme « a novel instrument for the evaluation of scientific journal that may challenge the established premiership of the journal IF in ranking scientific journals ».

López-Illescas et al. (2008) ont comparé les deux bases SCOPUS et WoS (= JCR) par rapport à leur couverture dans le domaine de la cancérologie. Conclusion : le WoS contient une sélection de revues avec le plus grand nombre de citations; mais presque toutes ces revues se trouvent aussi dans SCOPUS dont la couverture est plus large, avec des critères de sélection plus exhaustifs. L'analyse de Gorraiz \& Schloegl (2008) dans le domaine de la pharmacologie aboutit au même résultat : SCOPUS inclut un nombre significatif de revues («medium impact journals ») qui ne font pas partie de la sélection du JCR.

Le but de notre étude est d'appliquer la comparaison de Falagas et al. (2008a) aux des titres français ${ }^{7}$. L'objectif est de répondre à trois questions :

1. Quelle est la couverture pour les titres français indexés par Thomson Scientific et Elsevier, en termes de nombre de revues et domaines scientifiques couverts ?

2. Quelles sont les différences des deux indicateurs IF et SJR par rapport à l'échantillon des titres français?

3. Quel est l'intérêt de ce nouvel indicateur pour l'évaluation, en termes de représentativité des titres français?

\section{(2) Méthodologie}

La collecte des données empiriques a été effectuée en juillet 2008, en une seule fois.

L'interrogation des deux bases de données JCR Science Edition 2007 pour les domaines des sciences, technologies et médecine (STM) et JCR Social Sciences Edition 2007 pour les

\footnotetext{
${ }^{6} \mathrm{Cf}$. http://www.scimagojr.com/

${ }^{7}$ En fait, qu'est-ce qu'une revue française ? Cf. plus loin, la discussion des résultats.
} 
domaines des sciences humaines et sociales (SHS) a été réalisée via l'abonnement du CNRS au Web of Science de Thomson Scientific, sur le site du portail BiblioCNRS ${ }^{8}$.

Les titres français ont été sélectionnés à partir de l'indexation du pays d'édition (France) par l'ISI.

Les données exportées vers un fichier Excel et utilisées pour l'étude comparative ont été le titre abrégé de la revue, l'ISSN, le facteur d'impact IF, le nombre d'articles 2007 et le nombre de citations 2007.

L'extraction du Journal Rank Indicator a été réalisée via le site web de SCImago. Les titres français ont été sélectionnés à partir de l'indexation du pays d'édition (France) dans la base SCOPUS.

Les données exportées vers un fichier Excel et utilisées pour l'étude comparative ont été le titre abrégé de la revue, l'ISSN, le SJR, le nombre d'articles 2007, le nombre d'articles des trois dernières années, le nombre de citations des trois dernières années.

Les fichiers Excel JCR et SCImago ont été intégrés dans une base ACCESS.

Pour caractériser l'échantillon et comparer les deux extractions, d'autres informations issues de la base Ulrichsweb de CSA Proquest ont été ajoutées, notamment pour le domaine scientifique (discipline), l'éditeur de la revue et le pays d'édition.

\section{(3) Résultats}

Le $3^{\mathrm{e}}$ chapitre présente les résultats de l'analyse de l'échantillon sous trois aspects : Comment les revues se répartissent-elles entre SCOPUS et $J C R$, et quelle est la couverture des disciplines et domaines scientifiques par les deux concurrents (3.1.) ? Quelle est la différence entre l'indexation des titres français par le facteur d'impact de Thomson Scientific et le Journal Rank Indicator de SCImago (3.2.) ? Et quel est l'intérêt des deux produits pour les publications françaises en termes de représentativité (3.3) ?

\subsection{L'échantillon des titres français}

Le nombre de revues de l'échantillon, indexées par Thomson Scientific (JCR ) et/ou Elsevier (SCOPUS), s'élève à 368 titres. 166 revues sont indexées par Thomson Scientific (45\%), 345 revues par Elsevier (94\%), 143 revues appartiennent aux deux bases de données (39\%, cf. figure 1).

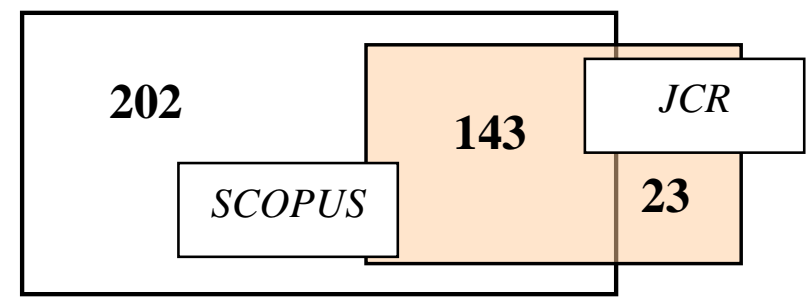

\footnotetext{
${ }^{8}$ http://bibliocnrs.inist.fr (visité en mars 2009)
} 
Figure 1 : Nombre de revues dans les deux bases de données SCOPUS et JCR

202 titres français sont indexés exclusivement par SCOPUS tandis que 23 titres se trouvent uniquement dans le $J C R$.

$41 \%$ des titres français de SCOPUS sont également indexés dans le JCR tandis que $86 \%$ des titres du JCR font partie de SCOPUS. La comparaison avec les chiffres de Gavel \& Iselid (2008) pour l'ensemble des titres $(=54 \%$ et $84 \%$ ) laisse penser que pour les titres français, la couverture de SCOPUS est relativement meilleure que pour l'ensemble des titres.

De cet échantillon de 368 revues, $82 \%$ sont issus des domaines STM et $18 \%$ des domaines SHS. (cf. figure 2).

\begin{tabular}{|l|c|c|c|c|c|c|}
\hline & \multicolumn{2}{|c|}{ JCR } & \multicolumn{2}{c|}{ SCOPUS } & \multicolumn{2}{c|}{ Echantillon } \\
\hline & Nb revues & en \% & Nb revues & en \% & Nb revues & en \% \\
\hline STM & 142 & $84 \%$ & 283 & $82 \%$ & 302 & $82 \%$ \\
\hline SHS & 24 & $16 \%$ & 62 & $18 \%$ & 66 & $18 \%$ \\
\hline Total & 166 & $100 \%$ & 345 & $100 \%$ & 368 & $100 \%$ \\
\hline
\end{tabular}

Figure 2 : Répartition des revues par grand domaine STM et SHS

Les différences entre les bases de données concurrentes sont plus visibles au niveau des disciplines scientifiques (cf. figure 3 et 4).

Dans les disciplines STM, SCOPUS indexe deux fois plus de titres que le JCR (cf. figure 3).

\begin{tabular}{|l|c|c|c|c|c|c|}
\hline & \multicolumn{2}{|c|}{ JCR } & \multicolumn{2}{c|}{ SCOPUS } & \multicolumn{2}{c|}{ Echantillon } \\
\hline & Nb revues & en \% & Nb revues & en \% & Nb revues & en \% \\
\hline $\begin{array}{l}\text { Sciences } \\
\text { médicales }\end{array}$ & 47 & $33 \%$ & 160 & $57 \%$ & 162 & $54 \%$ \\
\hline $\begin{array}{l}\text { Sciences du } \\
\text { Vivant }\end{array}$ & 33 & $23 \%$ & 39 & $14 \%$ & 45 & $15 \%$ \\
\hline $\begin{array}{l}\text { Sciences de } \\
\text { l'Univers et de } \\
\text { la Matière }\end{array}$ & 22 & $15 \%$ & 25 & $9 \%$ & 30 & $10 \%$ \\
\hline Technologies & 20 & $14 \%$ & 23 & $8 \%$ & 27 & $9 \%$ \\
\hline Pharmacologie & 2 & $1 \%$ & 13 & $5 \%$ & 14 & $5 \%$ \\
\hline Mathématiques & 12 & $8 \%$ & 13 & $5 \%$ & 14 & $5 \%$ \\
\hline Divers & 6 & $4 \%$ & 10 & $4 \%$ & 10 & $3 \%$ \\
\hline Total & 142 & $100 \%$ & 283 & $100 \%$ & 302 & $100 \%$ \\
\hline
\end{tabular}

Figure 3 : Répartition des revues par disciplines scientifiques (STM)

Pour toutes les disciplines scientifiques, la couverture des revues françaises de la base SCOPUS est supérieure à celle du $J C R$, même si cette différence est plutôt marginale et se situe entre 1 et 11 titres dans les différents domaines, à l'exception des sciences médicales dont la couverture très large - 160 titres, comparé aux 47 titres des JCR - constitue la vraie particularité de SCOPUS. 
En SHS, l'analyse de l'échantillon de revues révèle le même schéma, avec 2,5 fois plus de revues dans SCOPUS que dans le JCR. De nouveau, la couverture de SCOPUS est plus large dans tous les domaines sauf pour l'histoire et l'archéologie qui sont représentés par quatre titres dans les deux bases. Contrairement aux disciplines en STM, il n'y a aucun domaine dominant ni aucune véritable particularité pour l'une ou l'autre des deux bases (cf. figure 4).

\begin{tabular}{|l|c|c|c|c|c|c|}
\hline & \multicolumn{2}{|c|}{ JCR } & \multicolumn{2}{c|}{ SCOPUS } & \multicolumn{2}{c|}{ Echantillon } \\
\hline & Nb revues & En \% & Nb revues & en \% & Nb revues & en \% \\
\hline $\begin{array}{l}\text { Psychologie, } \\
\text { éducation, } \\
\text { travail social }\end{array}$ & 4 & $17 \%$ & 10 & $16 \%$ & 11 & $17 \%$ \\
\hline $\begin{array}{l}\text { Sociologie, } \\
\text { anthropologie }\end{array}$ & 5 & $21 \%$ & 9 & $15 \%$ & 10 & $15 \%$ \\
\hline Géographie & 1 & $4 \%$ & 10 & $16 \%$ & 10 & $15 \%$ \\
\hline $\begin{array}{l}\text { Sciences } \\
\text { économiques }\end{array}$ & 3 & $13 \%$ & 8 & $13 \%$ & 8 & $12 \%$ \\
\hline $\begin{array}{l}\text { Sciences } \\
\text { politiques }\end{array}$ & 2 & $8 \%$ & 7 & $11 \%$ & 7 & $11 \%$ \\
\hline $\begin{array}{l}\text { Histoire, } \\
\text { archéologie }\end{array}$ & 4 & $17 \%$ & 4 & $6 \%$ & 5 & $8 \%$ \\
\hline Linguistique & 0 & $0 \%$ & 5 & $8 \%$ & 5 & $8 \%$ \\
\hline Santé publique & 2 & $8 \%$ & 5 & $8 \%$ & 5 & $8 \%$ \\
\hline Divers & 3 & $13 \%$ & 4 & $6 \%$ & 5 & $8 \%$ \\
\hline Total & 24 & $100 \%$ & 62 & $100 \%$ & 66 & $100 \%$ \\
\hline
\end{tabular}

Figure 4 : Répartition des revues par disciplines scientifiques (SHS)

23 titres du JCR ne font pas parti de la couverture de SCOPUS, 19 revues en STM et 4 en SHS, dont 5 titres publiés par EDP Sciences et 4 titres par Elsevier France ou Masson.

Parmi tous ces titres, plusieurs ont été créés avant 1850, dont le Journal de Mathématiques Pures et Appliquées, le Bulletin de la Société géologique de France et les Annales de la Société entomologique de France. D'après la base Ulrichsweb, 70\% des titres de l'échantillon ont une version électronique, et plus de la moitié sélectionnent leurs articles par une procédure d'évaluation par les pairs («peer review»).

Trois cent quatorze revues sont également indexées par les bases de données PASCAL et FRANCIS de l'Institut de l'Information Scientifique et Technique (INIST) du CNRS. La plupart des titres sont publiés en français mais environ 50 revues ont des titres anglais.

\subsection{Les indicateurs IF et SJR des titres français}

En comparaison avec l'indicateur SJR issu de SCOPUS, l'indicateur IF du JCR se répartit sur un panel plus élargi de valeurs. Le JCR distribue les 166 revues avec IF sur 155 rangs différents. La valeur minimale est de 0,009 pour la revue Alcatel Telecommunications Review, la valeur maximale est de 4,259 pour la revue Astronomy and Astrophysics (cf. figure 5). 


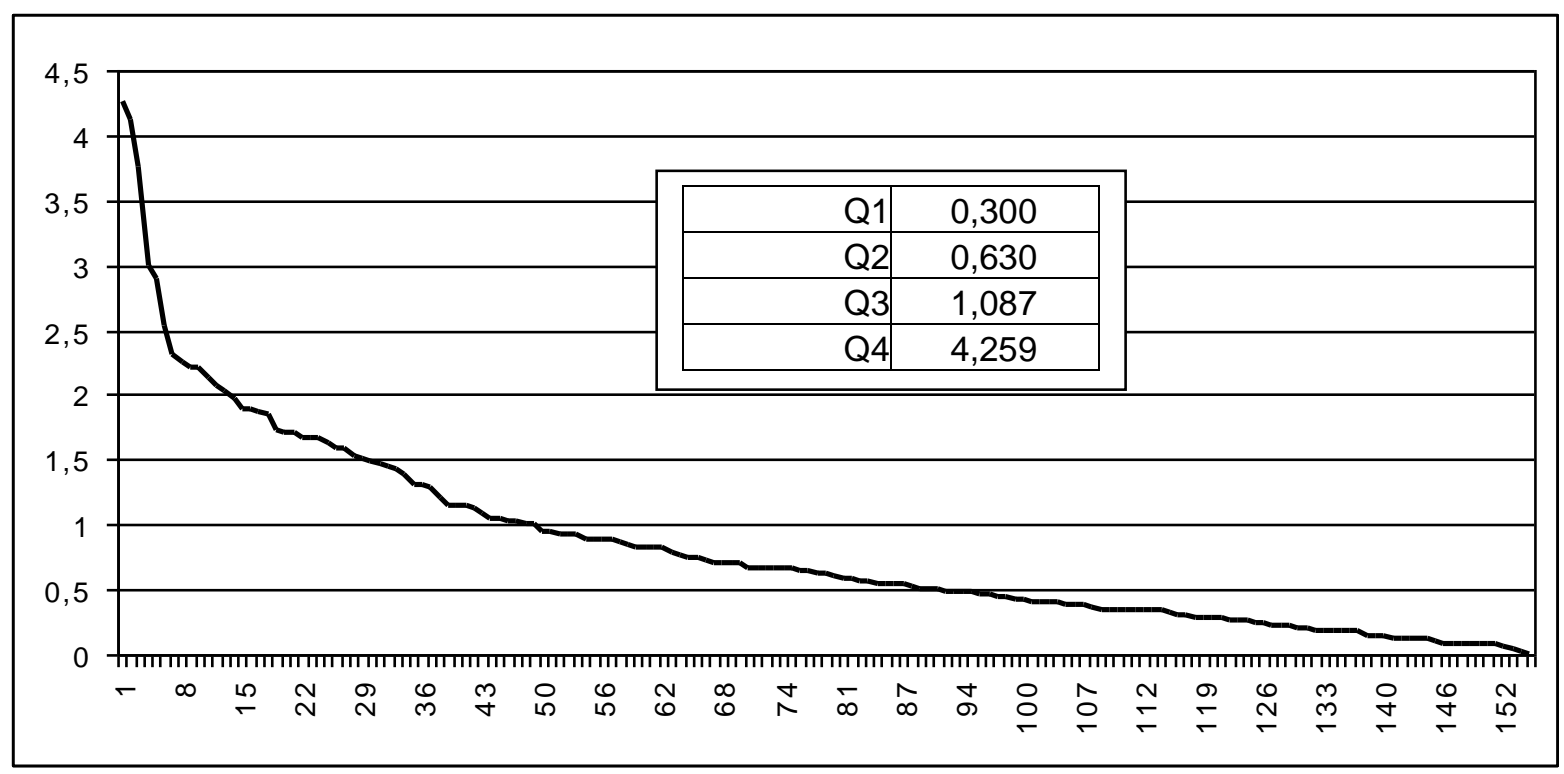

Figure 5 : Distribution des revues avec IF (JCR), avec valeurs des quartiles

Le classement des 345 revues en fonction du SJR s'étend sur seulement 89 rangs. La valeur minimale est de 0,00 pour 20 revues, la valeur maximale est de 1,14 pour la revue Biology of the Cell (cf. figure 6).

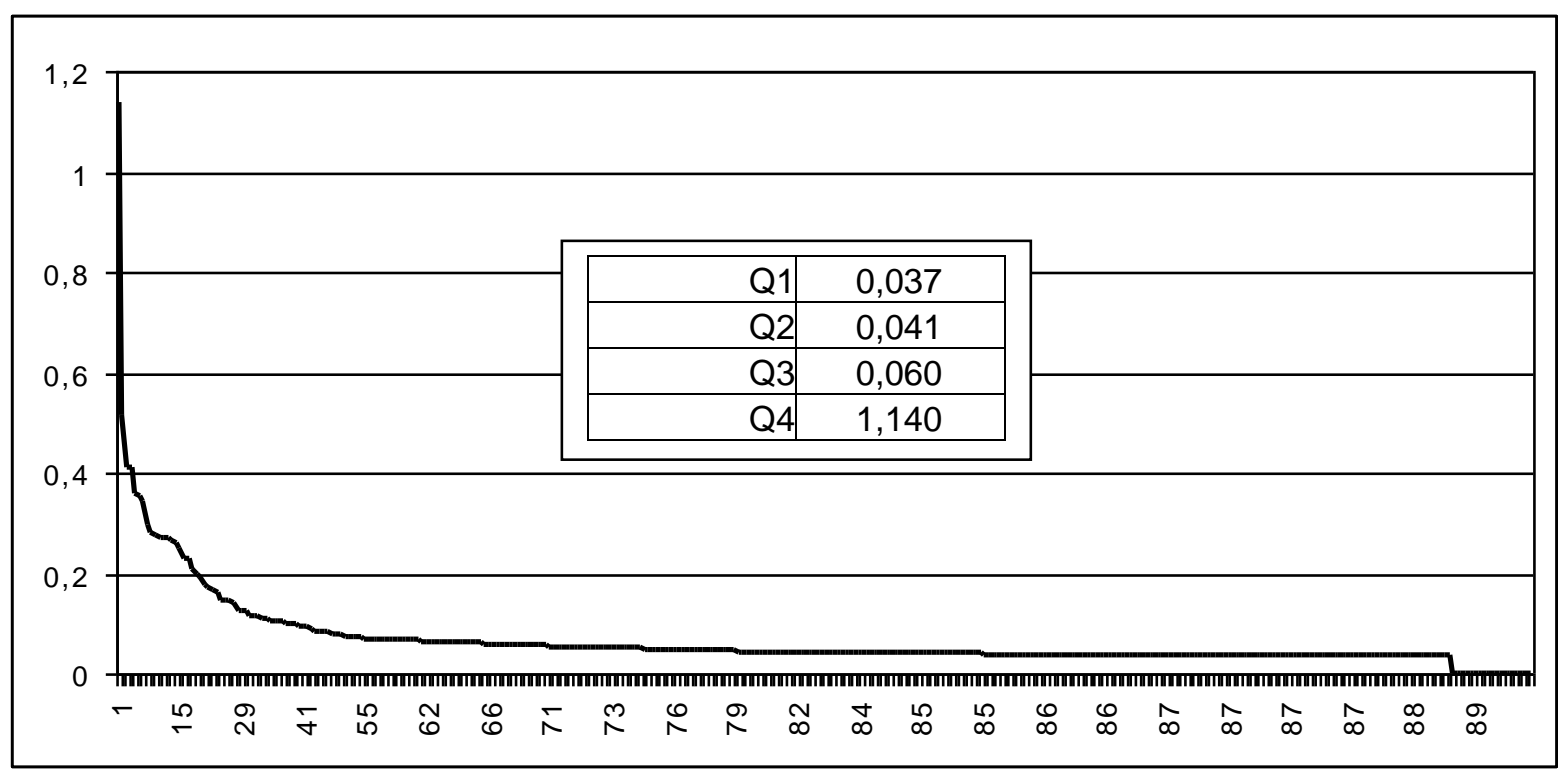

Figure 6 : Distribution des revues avec SJR (SCOPUS), avec valeurs des quartiles

Visiblement, le facteur d'impact du JCR différencie mieux les revues que le SJR. Malgré cette différence, les deux indicateurs ont une forte corrélation. Afin d'illustrer la relation entre les deux indicateurs, nous avons établi un nuage de points des 143 revues françaises avec IF et SJR (cf. figure 7). 


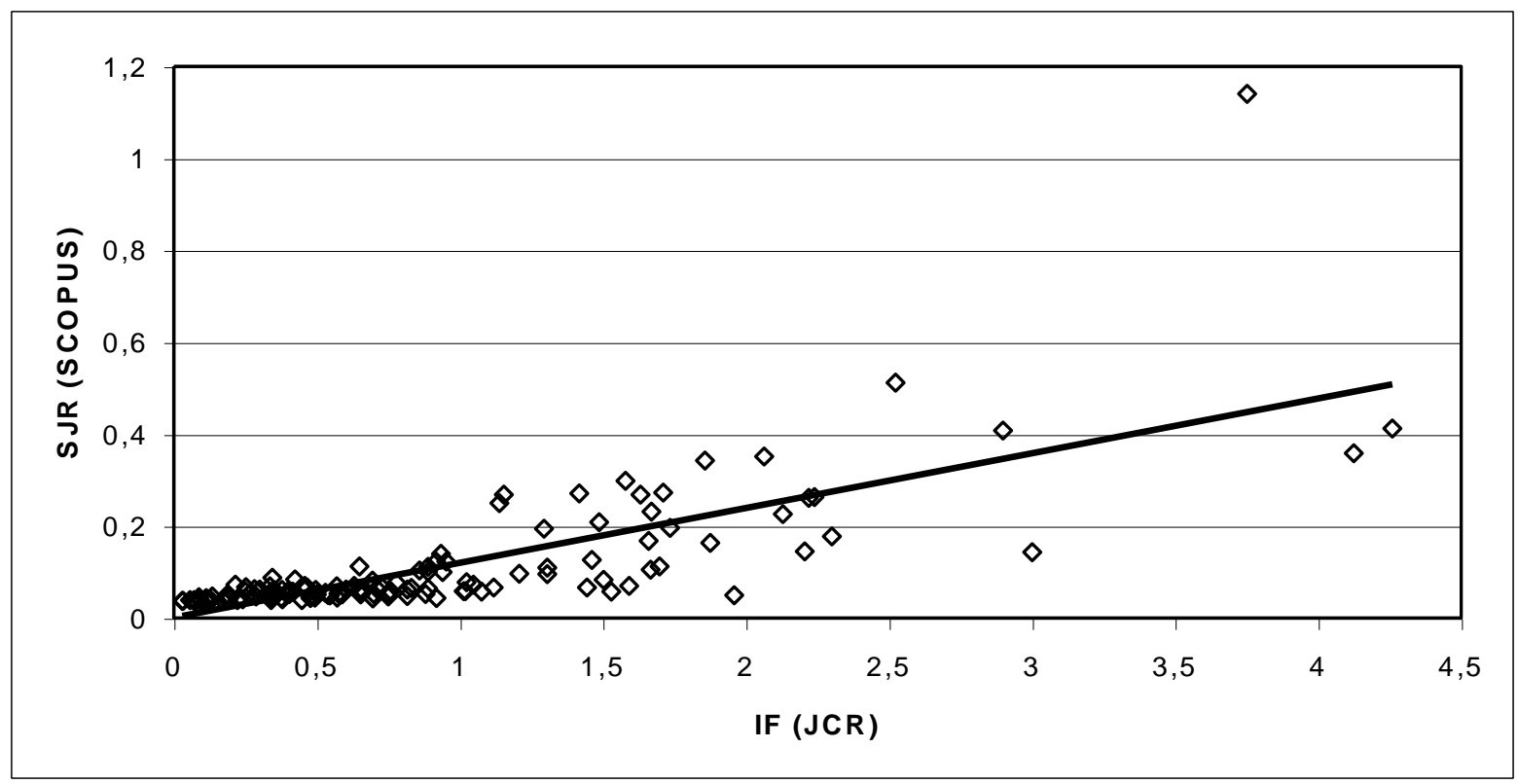

Figure 7 : Relation entre IF et SJR (avec courbe de tendance linéaire)

Pour ces revues, la corrélation entre les deux indicateurs IF et SJR est significative et s'élève à 0,76. La revue avec un IF $=3,75$ et un SJR $=1,14$ est Biology of the Cell de Portland Press.

\subsubsection{Comparaison du classement par IF et SJR (ranking)}

Au sein des 143 titres communs aux deux bases de données, le classement du JCR est plus favorable pour 34 titres. La différence la plus importante est enregistrée pour le ESA Bulletin qui occupe la $14^{\mathrm{e}}$ place dans le $J C R$, alors qu'il est à la $75^{\mathrm{e}}$ place dans SCOPUS. Deux titres, à savoir Genetics Selection Evolution et Aquatic Living Resources, occupent le même rang, quel que soit le classement, se retrouvant respectivement à la $19^{\mathrm{e}}$ et $60^{\mathrm{e}}$ place. Le classement de SCOPUS est plus avantageux pour 107 titres. Le gain le plus important est repéré pour le titre Journal des Maladies Vasculaires qui passe de la $129^{\mathrm{e}}$ place dans le $J C R$ à la $53^{\mathrm{e}}$ dans SCOPUS.

La comparaison du classement pour l'ensemble de l'échantillon (368 revues) donne le résultat suivant. Dans le classement des vingt premiers titres du JCR se retrouvent 12 titres classés parmi les vingt premiers de SCOPUS et vice-versa (cf. figures 8 et 9).

\begin{tabular}{|c|r|l|r|c|}
\hline $\begin{array}{c}\text { Rang } \\
\text { IF }\end{array}$ & \multicolumn{1}{c|}{ IF } & \multicolumn{1}{c|}{ Titre } & RJR & $\begin{array}{c}\text { Rang } \\
\text { SJR }\end{array}$ \\
\hline $\mathbf{1}$ & 4,259 & Astronomy and Astrophysics & 0,412 & 3 \\
\hline $\mathbf{2}$ & 4,125 & Veterinary Research & 0,358 & 5 \\
\hline $\mathbf{3}$ & 3,752 & Biology of the Cell & 1,14 & 1 \\
\hline $\mathbf{4}$ & 3 & Geostandards and Geoanalytical Research & 0,143 & 26 \\
\hline $\mathbf{5}$ & 2,899 & Biochimie & 0,408 & 4 \\
\hline $\mathbf{6}$ & 2,523 & Microbes and Infection & 0,512 & 2 \\
\hline $\mathbf{7}$ & 2,301 & European Journal of Medicinal Chemistry & 0,177 & 21 \\
\hline $\mathbf{8}$ & 2,24 & International Journal of Tuberculosis and Lung Disease & 0,263 & 13 \\
\hline $\mathbf{9}$ & 2,219 & Research in Microbiology & 0,261 & 14 \\
\hline
\end{tabular}




\begin{tabular}{|c|r|l|c|c|}
$\mathbf{1 0}$ & 2,206 & Europhysics Letters & 0,145 & 25 \\
\hline $\mathbf{1 1}$ & 2,129 & Fundamental \& Clinical Pharmacology & 0,226 & 17 \\
\hline $\mathbf{1 2}$ & 2,064 & European Cytokine Network & 0,351 & 6 \\
\hline $\mathbf{1 3}$ & 2,025 & European Physical Journal E. Soft Matter & & \\
\hline $\mathbf{1 4}$ & 1,959 & ESA Bulletin & 0,049 & 75 \\
\hline $\mathbf{1 5}$ & 1,896 & Chemical Senses & & \\
\hline $\mathbf{1 6}$ & 1,895 & Reproduction, Nutrition, Development & 0,163 & 24 \\
\hline $\mathbf{1 7}$ & 1,875 & European Psychiatry & 0,342 & 7 \\
\hline $\mathbf{1 8}$ & 1,857 & European Journal of Medical Genetics & 0,196 & 19 \\
\hline $\mathbf{1 9}$ & 1,735 & Genetics Selection Evolution & 0,273 & 10 \\
\hline $\mathbf{2 0}$ & 1,71 & Diabetes \& Metabolism & \\
\hline
\end{tabular}

Figure 8 : Classement des vingt premiers titres avec IF (JCR)

Trois titres avec IF - European Physical Journal E Soft Matter, Chemical Senses et Reproduction, Nutrition, Development - ne font pas parti de la base SCOPUS.

Le classement des vingt premiers titres selon SCOPUS détient un titre sans facteur d'impact, à savoir The Cancer Journal (Le Journal du Cancer), dont la parution en version imprimée a été arrêtée en 1998 (cf. figure 9).

\begin{tabular}{|c|r|l|r|c|}
\hline $\begin{array}{c}\text { Rang } \\
\text { IF }\end{array}$ & \multicolumn{1}{|c|}{ IF } & \multicolumn{1}{c|}{ Titre } & SJR & $\begin{array}{c}\text { Rang } \\
\text { SJR }\end{array}$ \\
\hline 3 & 3,752 & Biology of the Cell & 1,14 & $\mathbf{1}$ \\
\hline 6 & 2,523 & Microbes and Infection & 0,512 & $\mathbf{2}$ \\
\hline 1 & 4,259 & Astronomy and Astrophysics & 0,412 & $\mathbf{3}$ \\
\hline 5 & 2,899 & Biochimie & 0,408 & $\mathbf{4}$ \\
\hline 2 & 4,125 & Veterinary Research & 0,358 & $\mathbf{5}$ \\
\hline 12 & 2,064 & European Cytokine Network & 0,351 & $\mathbf{6}$ \\
\hline 18 & 1,857 & European Journal of Medical Genetics & 0,342 & $\mathbf{7}$ \\
\hline 27 & 1,579 & Journal of Physiology & 0,298 & $\mathbf{8}$ \\
\hline & & The Cancer Journal & 0,281 & $\mathbf{9}$ \\
\hline 20 & 1,71 & Diabetes \& Metabolism & 0,273 & $\mathbf{1 0}$ \\
\hline 33 & 1,418 & Biomedicine \& Pharmacotherapy & 0,271 & $\mathbf{1 1}$ \\
\hline 25 & 1,632 & Comptes rendus. Biologies & 0,268 & $\mathbf{1 2}$ \\
\hline 39 & 1,154 & Cellular and Molecular Biology & 0,268 & $\mathbf{1 2}$ \\
\hline 8 & 2,24 & International Journal of Tuberculosis and Lung Disease & 0,263 & $\mathbf{1 3}$ \\
\hline 9 & 2,219 & Research in Microbiology & 0,261 & $\mathbf{1 4}$ \\
\hline 41 & 1,138 & Transfusion Clinique et Biologique & 0,25 & $\mathbf{1 5}$ \\
\hline 22 & 1,669 & Plant Physiology and Biochemistry & 0,231 & $\mathbf{1 6}$ \\
\hline 11 & 2,129 & Fundamental \& Clinical Pharmacology & 0,226 & $\mathbf{1 7}$ \\
\hline 30 & 1,487 & Apidologie & 0,208 & $\mathbf{1 8}$ \\
\hline 19 & 1,735 & Genetics Selection Evolution & 0,196 & $\mathbf{1 9}$ \\
\hline 37 & 1,294 & EJD. European Journal of Dermatology & 0,194 & $\mathbf{2 0}$ \\
\hline
\end{tabular}

Figure 9 : Classement des vingt premiers titres avec SJR (SCOPUS)

Comparé aux vingt premiers titres avec IF, ce dernier tableau contient davantage de revues avec un titre français. Comme pour le $J C R$, ces vingt premiers titres appartiennent tous aux différents domaines STM. 


\subsubsection{Le cas des revues en SHS}

Puisque le classement des vingt meilleurs titres avec IF ou SJR ne contient pas de revues en SHS, nous avons établi les mêmes tableaux uniquement pour les revues issues des différents domaines des sciences humaines et sociales ou des arts. Voici le résultat, d'abord pour les 20 titres avec le facteur d'impact (IF) le plus élevé (cf. figure 10).

\begin{tabular}{|c|r|l|r|c|}
\hline Rang IF & \multicolumn{1}{|c|}{ IF } & \multicolumn{1}{|c|}{ Titre } & SJR & Rang SJR \\
\hline $\mathbf{5 5}$ & 0,889 & Comptes rendus. Palevol & 0,111 & 32 \\
\hline $\mathbf{5 7}$ & 0,88 & Comptes rendus. Géoscience & 0,052 & 72 \\
\hline $\mathbf{7 2}$ & 0,658 & Geobios & 0,061 & 64 \\
\hline $\mathbf{8 0}$ & 0,603 & Journal of Cultural Heritage & 0,062 & 63 \\
\hline $\mathbf{8 4}$ & 0,57 & Revue d'Epidémiologie et de Santé Publique & 0,069 & 56 \\
\hline $\mathbf{9 8}$ & 0,448 & Travail Humain & 0,039 & 85 \\
\hline $\mathbf{1 0 1}$ & 0,407 & Sciences Sociales et santé & 0,056 & 69 \\
\hline $\mathbf{1 0 5}$ & 0,38 & L'Année Psychologique & 0,041 & 83 \\
\hline $\mathbf{1 1 1}$ & 0,345 & L'Anthropologie & 0,087 & 43 \\
\hline $\mathbf{1 1 2}$ & 0,34 & Sociologie du Travail & 0,039 & 85 \\
\hline $\mathbf{1 1 4}$ & 0,333 & Annales de Paléontologie & & \\
\hline $\mathbf{1 1 6}$ & 0,3 & Population & 0,062 & 63 \\
\hline $\mathbf{1 2 1}$ & 0,272 & International Journal of Psychology & & \\
\hline $\mathbf{1 2 2}$ & 0,269 & Archives Européennes de Sociologie & 0,039 & 85 \\
\hline $\mathbf{1 2 8}$ & 0,222 & Revue Française de Sociologie & 0,038 & 86 \\
\hline $\mathbf{1 3 7}$ & 0,171 & Revue de Géographie aApine & & \\
\hline $\mathbf{1 3 9}$ & 0,138 & International Social Science Journal & 0,037 & 87 \\
\hline $\mathbf{1 4 3}$ & 0,119 & Mouvement Social & 0,037 & 87 \\
\hline $\mathbf{1 4 4}$ & 0,113 & Revue d'Economie Politique & 0,037 & 87 \\
\hline $\mathbf{1 4 5}$ & 0,093 & L'Homme & & \\
\hline
\end{tabular}

Figure 10 : Classement des vingt premiers titres SHS avec IF (JCR)

De ces 20 revues, quatre ne font pas partie des 20 revues les mieux classées en fonction du SJR (cf. figure 11), et quatre autres ne sont pas indexées dans SCOPUS (Annales de Paléontologie, International Journal of Psychology, Archives Européennes de Sociologie, International Social Science Journal). La figure 11, qui présente les 20 revues les mieux classées selon leur SJR, témoigne d'une relative stabilité parmi les revues SHS.

\begin{tabular}{|c|r|l|r|c|}
\hline Rang IF & \multicolumn{1}{|c|}{ IF } & \multicolumn{1}{|c|}{ Titre } & SJR & Rang SJR \\
\hline 55 & 0,889 & Comptes rendus. Palevol & 0,111 & $\mathbf{3 2}$ \\
\hline 111 & 0,345 & L'Anthropologie & 0,087 & $\mathbf{4 3}$ \\
\hline 84 & 0,57 & Revue d'Epidémiologie et de Santé Publique & 0,069 & $\mathbf{5 6}$ \\
\hline 80 & 0,603 & Journal of Cultural Heritage & 0,062 & $\mathbf{6 3}$ \\
\hline 116 & 0,3 & Population & 0,062 & $\mathbf{6 3}$ \\
\hline 72 & 0,658 & Geobios & 0,061 & $\mathbf{6 4}$ \\
\hline 101 & 0,407 & Sciences Sociales et Santé & 0,056 & $\mathbf{6 9}$ \\
\hline 57 & 0,88 & Comptes rendus. Géoscience & 0,052 & $\mathbf{7 2}$ \\
\hline & & Revue de Micropaléontologie & 0,052 & $\mathbf{7 2}$ \\
\hline
\end{tabular}




\begin{tabular}{|c|c|c|c|c|}
\hline & & $\begin{array}{l}\text { Cahiers de Sociologie et de Démographie } \\
\text { Médicales }\end{array}$ & 0,051 & 73 \\
\hline & & Psychologie Française & 0,05 & 74 \\
\hline & & Promotion \& Education & 0,047 & 77 \\
\hline & & European review of applied psychology & 0,044 & 80 \\
\hline \multirow[t]{4}{*}{105} & 0,38 & L'Année psychologique & 0,041 & 83 \\
\hline & & Santé Publique & 0,041 & 83 \\
\hline & & Annales Médico-psychologiques & 0,04 & 84 \\
\hline & & L'Espace Géographique & 0,04 & 84 \\
\hline 98 & 0,448 & Travail Humain & 0,039 & 85 \\
\hline 112 & 0,34 & Sociologie du Travail & 0,039 & 85 \\
\hline 128 & 0,222 & Revue Française de Sociologie & 0,039 & 85 \\
\hline
\end{tabular}

Figure 11 : Classement des vingt premiers titres SHS avec SJR (SCOPUS)

Douze titres figurent parmi les mieux classés aussi bien pour le JCR que pour SCOPUS dont notamment Comptes rendus Palevol ${ }^{9}$, Revue d'Epidémiologie et de Santé Publique, Journal of Cultural Heritage, Geobios, Sciences sociales et Santé et Comptes rendus Géoscience. Néanmoins, ce tableau contient aussi huit titres sans IF, comme par exemple la Revue de Micropaléontologie, La Psychologie Française ou encore les Annales Médicopsychologiques.

\subsection{La visibilité des revues françaises dans les deux bases de données}

Ulrichsweb, le catalogue le plus complet des périodiques, contient pour la France 1795 revues vivantes à caractère scientifique dont 749 en STM et 1046 en SHS (chiffres de mars 2009). Parmi ces 1795 revues, $9 \%$ se retrouvent dans le JCR et 19\% dans SCOPUS.

Néanmoins, les différences entre les domaines scientifiques sont importantes. Le JCR couvre $19 \%$ des titres en STM tandis que SCOPUS contient le double, $38 \%$ (cf. annexe 1).

Le JCR couvre entre $13 \%$ et $35 \%$ des revues scientifiques françaises dans les différents domaines. Cette représentativité est relativement équilibrée, avec une légère préférence pour les sciences du vivant, les sciences appliquées (technologies) et les mathématiques.

SCOPUS est bien plus hétérogène, avec une représentativité par domaine entre $22 \%$ et $93 \%$. Mais on distingue très clairement deux groupes de disciplines : d'une part, les sciences médicales et du vivant, y compris la pharmacologie, dont presque la moitié des revues françaises (46\%) sont indexées par SCOPUS. De l'autre côté, les autres disciplines STM avec une couverture entre $20 \%$ et $30 \%$.

La situation pour les revues en SHS qui constituent la majeure partie de l'édition scientifique en France est complètement différente (cf. annexe 2). Certains domaines comme la littérature, la religion, la philosophie ou la linguistique ne sont pas ou peu indexés par le JCR et/ou SCOPUS. Ensemble ils représentent presque le tiers de l'édition SHS en France. Pour d'autres la représentativité est limitée, avec des taux entre $2 \%$ et $5 \%$ pour le $J C R$ et entre $2 \%$ et $12 \%$

\footnotetext{
${ }^{9}$ La série Palevol des Comptes Rendus de l'Académie des Sciences, une revue sur la paléontologie publiée chez Elsevier Masson, occupe la $1^{\mathrm{e}}$ position aussi bien du classement du JCR que de SCOPUS.
} 
pour SCOPUS. Avec deux exceptions : quelques revues SHS en santé publique (71\% de ces revues se retrouvent dans SCOPUS, 29\% dans le $J C R$ ), et des titres en géographie (40\% dans SCOPUS). Mais globalement, le taux de couverture des revues françaises en SHS est faible pour les deux bases, et la différence entre $2 \%$ pour le JCR et $6 \%$ pour SCOPUS ne paraît pas significative.

Pour mieux illustrer la représentativité (ou l'absence de représentativité) des deux bases par rapport à l'édition française de revues scientifiques, nous avons étudié un échantillon de 12 éditeurs dont l'offre correspond à 25-30\% du marché des revues en France (cf. figure 12).

\begin{tabular}{|c|c|c|c|c|c|c|}
\hline & \multicolumn{2}{|c|}{$J C R$} & \multicolumn{2}{|c|}{ SCOPUS } & \multicolumn{2}{|c|}{ Ulrichsweb (France) } \\
\hline & Nb revues & en \% & Nb revues & En \% & Nb revues & en \% \\
\hline Armand Colin & 3 & $10 \%$ & 6 & $21 \%$ & 29 & $100 \%$ \\
\hline CNRS $^{10}$ & 0 & $0 \%$ & 0 & $0 \%$ & 78 & $100 \%$ \\
\hline $\begin{array}{l}\text { Collège de } \\
\text { France }^{11}\end{array}$ & 1 & $5 \%$ & 2 & $10 \%$ & 21 & $100 \%$ \\
\hline EDP Sciences & 7 & $20 \%$ & 23 & $66 \%$ & 35 & $100 \%$ \\
\hline Elsevier $^{12}$ & 68 & $33 \%$ & 138 & $67 \%$ & 206 & $100 \%$ \\
\hline Erès & 0 & $0 \%$ & 0 & $0 \%$ & 25 & $100 \%$ \\
\hline John Libbey $^{13}$ & 8 & $25 \%$ & 23 & $72 \%$ & 32 & $100 \%$ \\
\hline Lavoisier & 5 & $25 \%$ & 7 & $35 \%$ & 20 & $100 \%$ \\
\hline L'Harmattan & 0 & $0 \%$ & 0 & $0 \%$ & 65 & $100 \%$ \\
\hline Peeters $^{14}$ & 0 & $0 \%$ & 0 & $0 \%$ & 25 & $100 \%$ \\
\hline PUF & 1 & $2 \%$ & 5 & $11 \%$ & 46 & $100 \%$ \\
\hline Springer $^{15}$ & 3 & $8 \%$ & 13 & $34 \%$ & 38 & $100 \%$ \\
\hline
\end{tabular}

Figure 12 : Représentativité des bases par éditeurs (exemples)

D'après ces chiffres, cinq éditeurs - Elsevier France (y compris Masson), Springer France, EDP Sciences, John Libbey et Lavoisier - sont relativement bien indexés dans les deux bases, et en particulier dans SCOPUS. Les revues d'autres éditeurs par contre, comme Armand Colin, L'Harmattan, Peeters ou PUF, ne s'y trouvent peu ou pas du tout.

Ajoutons que parmi les éditeurs (750 environ) de revues scientifique françaises, seuls $10 \%$ éditent des revues avec un IF (JCR) tandis que $17 \%$ publient des revues avec un SJR (SCOPUS) ; à peine $7 \%$ des éditeurs présentent des revues identifiées par les deux indicateurs. Autrement dit: la plus grande partie des éditeurs de revues françaises, dont beaucoup de sociétés savantes, laboratoires, presses universitaires etc., n'ont aucune visibilité dans les deux grandes bases bibliométriques.

\section{(4) Discussion}

\footnotetext{
${ }^{10}$ Co-éditeur de revues publiées par des équipes scientifiques

${ }^{11}$ Co-éditeur avec d'autres organismes (EHESS etc.)

${ }^{12}$ Elsevier France, Masson

${ }^{13}$ Editeur anglais avec une filiale en France (John Libbey Eurotext)

${ }^{14}$ Editeur belge avec bureau (antenne) à Paris

${ }^{15}$ Springer France
} 
Résumons les résultats de notre étude qui portait sur 368 revues scientifiques françaises avec IF et/ou SJR :

Quelle est la couverture pour les titres français indexés par Thomson Scientific (JCR) et Elsevier (SCOPUS), en termes de nombre et domaines scientifiques : 166 revues sont indexées par Thomson Scientific (45\%), 345 revues par Elsevier (94\%), 143 revues par les deux (39\%). 86\% des titres avec IF (JCR) font partie de SCOPUS. 82\% des 368 revues sont issus des domaines STM, $18 \%$ des domaines SHS. La seule différence significative entre les deux bases est la meilleure couverture de SCOPUS en médecine et pharmacologie.

Quelles sont les différences des deux indicateurs IF (JCR) et SJR (SCOPUS) par rapport à l'échantillon des titres français : Pour les titres avec IF et SJR, la corrélation entre les deux indicateurs est significative $(0,76)$. En termes de classement (ranking), le IF différencie mieux les revues que le SJR (155 vs. 89 rangs). Par contre, du fait de la couverture plus exhaustive de SCOPUS, le SJR rend visible au niveau international davantage de titres.

Quel est l'intérêt de ce nouvel indicateur SJR par rapport au IF de Thomson Scientific, pour l'évaluation, en termes de représentativité des titres français : L'intérêt de SCOPUS et de son indicateur dérivé $S J R$ réside dans la couverture plus représentative de l'édition française (19\% vs. 9\% pour le JCR/IF), notamment en STM (38\% vs. 19\%), beaucoup moins en SHS (6\% vs. 2\%). Sont indexés surtout les titres de quelques grands éditeurs français ou internationaux ; la plupart des éditeurs français (80\%-90\%) n'ont aucun titre dans le JCR et/ou SCOPUS, même si de nouveau SCOPUS est plus représentatif (avec 17\% des éditeurs vs. $10 \%$ pour le $J C R)$.

L'analyse des titres français issus des fichiers du JCR et de SCOPUS, avec l'extraction des titres français répertoriés dans Ulrichsweb, pose un certain nombre de problèmes :

ISSN et titre des revues : L'échantillon a été établi à partir des titres et ISSN des deux bases $S C O P U S$ et JCR. Or, SCOPUS répertorie les titres d'après l'ISSN électronique, alors que le $J C R$ garde l'ISSN de la version papier (ex. Journal of Porphyrins and Phthalocyanines). L'écriture différente (variantes) pour le même titre pose un autre problème. Un exemple : identifié sous Mathematical Modelling and Numerical Analysis dans SCOPUS, la même revue figure sous ESAIM-MATH MODEL NUM dans le JCR.

Domaine scientifique : L'indexation des domaines scientifiques par le JCR, SCOPUS et Ulrichsweb n'est pas cohérente ou concordante. Sept titres de l'échantillon (dont Geobios, une revue en paléontologie) ont un facteur d'impact renseigné à partir de la Science Edition (STM) du JCR mais sont classés en SHS dans Ulrichsweb. A l'inverse, le titre Evolution Psychiatrique est classé en STM par Ulrichsweb, alors qu'il a un facteur d'impact extrait du JCR Social Sciences Edition (SHS).

Caractère scientifique des périodiques : L'indexation du caractère scientifique des revues (scholarly/academic) n'est pas cohérente non plus. Par exemple, la revue La Houille Blanche fait partie des bases JCR et SCOPUS mais est considérée comme «presse spécialisée » (Newspaper Trade) par Ulrichsweb. A l'inverse, on peut s'interroger sur l'indexation des collections scolaires des éditions Ellipses comme « scientifique » par Ulrichsweb.

Pays d'édition : Les incohérences d'indexation concernent aussi le pays d'édition. Par exemple, le $J C R$ indexe un seul pays tandis qu'Ulrichsweb indique plusieurs pays. Ces 
incohérences reflètent une réalité parfois complexe. Citons quelques cas. Biology of the Cell est publié par l'éditeur anglais Portland Press pour le compte de deux sociétés françaises (Société Française des Microscopies et Société de Biologie Cellulaire de France). La revue appartient à notre échantillon car SCOPUS la considère comme revue française. De l'autre côté, la base Ulrichsweb contient une trentaine de titres «édités en France » avec IF pour lesquels le JCR indexe un autre pays puisque la France n'est pas le siège social de l'éditeur commercial. Deux autres revues de Lavoisier, l'une en anglais (European Journal of Control), l'autre en français (Revue d'Intelligence Artificielle) font partie de l'échantillon ; en fait, elles sont publiées par une filiale de Lavoisier (Hermes Sciences Publishing Ltd.) à Londres. On peut continuer la liste, en parlant d'Elsevier (European Journal of Medicinal Chemistry), de Springer (European Physical Journal), de John Libbey (Bulletin du Cancer) ou encore de Peeters qui, à cause de son bureau à Paris est indexé par Ulrichsweb comme éditeur français et pas uniquement belge. Pour être cohérent avec les objectifs de notre analyse (comparaison des deux bases bibliométriques par rapport à leur intérêt pour les publications françaises, et non pas critique de leur indexation, qualité ou utilisation), en cas de doute ou divergence nous avons toujours privilégié l'indexation par SCOPUS et JCR. Néanmoins, la question de savoir ce qu'est réellement une revue scientifique française reste posée.

Ces problèmes, nous l'avons déjà dit, ne sont pas spécifiques à notre analyse, et ils ont déjà été décrits et étudiés par d'autres auteurs. (cf. par exemple Gavel \& Iselid 2008).

Ils sont liés aux différences de sélection et d'indexation des trois produits utilisés, aussi à leurs objectifs et positionnements divergents (bibliométrie, recherche d'information, gestion des abonnements etc.). Mais derrière ces incohérences se dessine aussi un paysage de l'édition scientifique hétérogène, complexe, dont les contours nationaux perdent de plus en plus de leur pertinence et intérêt. Pour les résultats de notre comparaison, ces problèmes introduisent un certain degré d'imprécision mais l'impact sur la qualité de l'analyse reste limité et maîtrisé.

\section{(5) Conclusion}

L'évaluation de la science, l'analyse scientifique de l'activité et de la production des chercheurs et établissements est une nécessité et en même temps, un enjeu politique. Le facteur d'impact (IF) des revues, développé par Garfield et commercialisé par Thomson Scientific, joue (toujours) un rôle central dans les dispositifs d'évaluation.

Or, les limites méthodologiques du FI sont connues. Notamment, la sur-représentation des revues anglophones. Malgré l'argument que l'anglais est la langue internationale de la science par excellence (cf. Moed 2005), le nombre restreint de revues avec IF publiées dans d'autres langues ${ }^{16}$ introduit un biais systématique en faveur des publications en anglais.

Dans ce contexte, notre analyse confirme l'intérêt du nouvel indicateur SJR comme alternative au FI. La principale raison : le nombre plus élevé de revues françaises indexées par SCOPUS qui sert de base au calcul du SJR. Depuis 2008, plus de 50 revues françaises ont été ajoutées à $S C O P U S$. L'intérêt du SJR va probablement encore accroître avec l'intégration supplémentaire de plus de 1000 revues en SHS dans SCOPUS, annoncée par Elsevier pour avril 2009.

\footnotetext{
${ }^{16}$ D'après la base Ulrichsweb, seulement $12 \%$ des revues scientifiques avec comité de lecture contiennent des articles écrits dans une autre langue que l'anglais.
} 
Certes, le SJR a ses propres faiblesses, comme par exemple son mode de calcul qui paraît assez (trop) compliqué (cf. Falagas et al. 2008b) ou encore l'utilisation de tous les articles et rubriques du sommaire pour le calcul de l'indicateur (y compris les lettres, synthèses, critiques, éditoriaux etc.) et non pas seulement des articles scientifiques, «citables », un biais qui baisse tendanciellement l'indicateur.

Cependant, notre objectif n'est pas de présenter les caractéristiques, forces et faiblesses des deux indicateurs en général ${ }^{17}$ et non plus, d'évaluer l'usage fait de ces indicateurs. Pour terminer notre analyse, nous nous contenterons donc de trois remarques.

Rapport entre IF et SJR : La corrélation entre les deux indicateurs est plutôt élevée, malgré leurs différences et spécificités. Dans la mesure où le calcul du SJR tient compte du prestige de la revue qui cite («qualité des citations ») et où les auto-citations sont éliminées du calcul, Falagas et al. (2008b) parlent du SJR comme «indicateur de qualité d'une revue », comparé au IF «indicateur de la popularité d'une revue». Cette proximité toute relative des deux indicateurs («expression of popularity») est confirmée par l'analyse statistique de 39 indicateurs par Bollen et al. (2009).

Alternative ou complémentarité : Cette dernière étude soulève d'ailleurs une autre question, à savoir la nature de l'impact d'une publication. En vu de leurs résultats, Bollen et al. (2009) sont convaincu : «scientific impact is a multi-dimensional construct ». Faut-il donc vraiment choisir entre IF et SJR ? Ne serait-il pas plus judicieux de continuer l'analyse des modes de calcul, biais, contenu, relation etc. et de construire une méthodologie d'évaluation multidimensionnelle, à partir de plusieurs indicateurs et en abandonnant l'idée que le IF soit le « golden standard» de l'impact scientifique?

Evolution des mesures d'impact : D'autres arguments parlent en faveur d'un tel changement de paradigme, dont l'émergence de nouveaux indicateurs d'usage à partir des ressources en ligne (usage factors) et une critique plus globale des analyses quantitatives de l'impact. Les contours d'un nouveau dispositif d'évaluation de l'impact se dessinent qui remplacera à terme les approches simplistes basées sur un ou deux indicateurs quantitatifs (IF, h-index etc.). ${ }^{18} \mathrm{Il}$ paraît pertinent et probable que le SJR de SCOPUS y trouve sa place.

\section{(6) Bibliographie}

Ball R., Tunger D. (2006): Science indicators revisited - Science citation index versus SCOPUS: A bibliometric comparison of both citation databases. Information Services and Use 26, 4, 293- 301.

Baudoin L., Haeffner-Cavaillon N., Pinhas N., Mouchet S., Kordon C. (2004) : Indicateurs bibliométriques : réalités, mythes et prospective. M/S Médecine Sciences, 20, 10, 909-915. http://www.erudit.org/revue/MS/2004/v20/n10/009339ar.html

Bollen J., Rodriguez M.A., Van de Sompel H. (2006) : Journal Status. Scientometrics 69, 669687.

Bollen J., Van de Sompel H., Hagberg A., Chute R. (2009) : A principal component analysis of 39 scientific impact measures. Preprint. 12 février 2009. http://arXiv:0902.2183v1

\footnotetext{
${ }^{17}$ Cf. pour cela le tableau comparatif chez Falagas et al. (2008b).

${ }^{18}$ Cf. Lefebvre 2008 ou Durand-Barthez 2008.
} 
de Moya-Anegón F., Chinchilla-Rodríguez Z., Vargas-Quesada B., Corera-Álvarez E., Muñoz-Fernández F.J., González-Molina A., Herrero-Solana V. (2007) : Coverage analysis of Scopus: A journal metric approach. Scientometrics 73, 1, 53-78.

Dong P., Loh M., Mondry A. (2005) : The «impact factor» revisited. Biomedical Digital Libraries 2, 7. http://www.bio-diglib.com/content/2/1/7

Durand-Barthez M. (2006): L'évaluation des publications scientifiques: du facteur d'impact à l'indice de notoriété. http://archivesic.ccsd.cnrs.fr/sic_00083870/fr/

Durand-Barthez M. (2008): Les nouvelles formes d'évaluation scientifique: quelles évolutions en sciences, technique et médecine ? http://archivesic.ccsd.cnrs.fr/sic_00260459/fr/

Falagas M.E., Kouranos V.D., Arencibia-Jorge R., Karageorgopoulos D.E. (2008a) : Comparison of SCImago journal rank indicator with journal impact factor. The FASEB Journal, 22, April 11, 1-6.

Falagas M.E., Pitsouni E.I., Malietzis G.A., Pappas G. (2008b) : Comparison of PubMed, Scopus, Web of Science, and Google Scholar: strengths and weaknesses. The FASEB Journal, 22, 338-342.

Garfield E. (1955) : Citation indexes for science. Science 122, 108-111.

Garfield E. (1972) : Citation analysis as a tool in journal evaluation. Science 178, 471-479.

Garfield E. (2006) : The history and meaning of the journal impact factor. JAMA 295, 90-93.

Gavel Y., Iselid L. (2008) : Web of Science and Scopus: a journal title overlap study. Online Information Review 32, 1, 8-21.

Gorraiz J., Schloegl C. (2008) : A bibliometric analysis of pharmacology and pharmacy journals: Scopus versus Web of Science. Journal of Information Science, 34, 5, 715-725.

Lefebvre M. (2008) : L'évaluation des savoirs scientifiques : modalités et enjeux. In Schöpfel J. (dir.) : La publication scientifique. Paris, Lavoisier.

López-Illescas C., de Moya-Anegón F., Moed H.F. (2008) : Coverage and citation impact of oncological journals in the Web of Science and Scopus. Journal of Informetrics 2, 304-316.

Magri M.H., Solari A., Rerat K. (1996): Les périodiques scientifiques d'audience internationale au travers du Journal Citation Reports : analyse du système d'évaluation de l'ISI. Application à l'étude de la production de l'INRA. L'information scientifique et technique: nouveaux enjeux documentaires et éditoriaux. Colloque INRA, 21-23 octobre 1996, Tours. http://www.inra.fr/internet/Unites/UCD_Jouy/scientom/magri_fr.htm

Moed H.E. (2005) : Citation analysis in research evaluation. Dordrecht, Springer.

Neuhaus C., Daniel H. (2008) : Data sources for performing citation analysis: An overview. Journal of Documentation 64, 2, 193-210.

Ojasoo T., Maisonneuve H., Matillon Y. (2002): Le facteur d'impact des revues, un indicateur bibliométrique à manier avec prudence. La Presse Médicale 31, 17, 775-781.

Page L., Brin S., Motwani R., Winograd T. (1998): The PageRank citation ranking : Bringing order to the web. Technical Report. Stanford Digital Library Technologies Project SIDL-WP-1999-0120.

Pinhas N., Kordon C. (1997) : Du bon usage du facteur d'impact. INSERM Actualités 154, 710. http://www.cindoc.csic.es/cybermetrics/pdf/578.pdf

Pocard M. (2002) : Le facteur d'impact des revues médicales : jugement de Salomon ou danse des sept voiles ? Annales de Chirurgie 52, 7, 595-597.

Tous les sites Web ont été visités en mars 2009.

\section{Annexe 1}

\begin{tabular}{|l|l|l|l|}
\hline & JCR & SCOPUS & Ulrichsweb (France) \\
\hline
\end{tabular}




\begin{tabular}{|l|c|c|c|c|c|c|}
\hline & Nb revues & en \% & Nb revues & en \% & Nb revues & en \% \\
\hline $\begin{array}{l}\text { Sciences } \\
\text { médicales }\end{array}$ & 47 & $13 \%$ & 160 & $45 \%$ & 352 & $100 \%$ \\
\hline $\begin{array}{l}\text { Sciences du } \\
\text { Vivant }\end{array}$ & 33 & $35 \%$ & 39 & $41 \%$ & 94 & $100 \%$ \\
\hline $\begin{array}{l}\text { Sciences de } \\
\text { l'Univers et de } \\
\text { la Matière }\end{array}$ & 22 & $19 \%$ & 25 & $22 \%$ & 115 & $100 \%$ \\
\hline Technologies & 20 & $24 \%$ & 23 & $27 \%$ & 84 & $100 \%$ \\
\hline Pharmacologie & 2 & $14 \%$ & 13 & $93 \%$ & 14 & $100 \%$ \\
\hline Mathématiques & 12 & $21 \%$ & 13 & $23 \%$ & 57 & $100 \%$ \\
\hline Divers & 6 & $16 \%$ & 10 & $27 \%$ & 37 & $100 \%$ \\
\hline Total & 142 & $19 \%$ & 283 & $38 \%$ & 749 & $100 \%$ \\
\hline
\end{tabular}

Représentativité des bases par disciplines scientifiques (STM)

\section{Annexe 2}

\begin{tabular}{|l|c|c|c|c|c|c|}
\hline & \multicolumn{2}{|c|}{ JCR } & \multicolumn{2}{c|}{ SCOPUS } & \multicolumn{2}{c|}{ Ulrichsweb (France) } \\
\hline & Nb revues & en \% & Nb revues & En \% & Nb revues & en \% \\
\hline $\begin{array}{l}\text { Psychologie, } \\
\text { éducation, } \\
\text { travail social }\end{array}$ & 4 & $4 \%$ & 10 & $9 \%$ & 106 & $100 \%$ \\
\hline $\begin{array}{l}\text { Sociologie, } \\
\text { anthropologie }\end{array}$ & 5 & $5 \%$ & 9 & $9 \%$ & 98 & $100 \%$ \\
\hline Géographie & 1 & $4 \%$ & 10 & $40 \%$ & 25 & $100 \%$ \\
\hline $\begin{array}{l}\text { Sciences } \\
\text { économiques, } \\
\text { droit }\end{array}$ & 3 & $3 \%$ & 8 & $9 \%$ & 91 & $100 \%$ \\
\hline $\begin{array}{l}\text { Sciences } \\
\text { politiques }\end{array}$ & 2 & $3 \%$ & 7 & $12 \%$ & 59 & $100 \%$ \\
\hline $\begin{array}{l}\text { Histoire, } \\
\text { archéologie }\end{array}$ & 4 & $2 \%$ & 4 & $2 \%$ & 208 & $100 \%$ \\
\hline Linguistique & 0 & $0 \%$ & 5 & $5 \%$ & 100 & $100 \%$ \\
\hline Santé publique & 2 & $29 \%$ & 5 & $71 \%$ & 7 & $100 \%$ \\
\hline $\begin{array}{l}\text { Arts, } \\
\text { littérature }\end{array}$ & 0 & $0 \%$ & 0 & $0 \%$ & 178 & $100 \%$ \\
\hline Religion & 0 & $0 \%$ & 0 & $0 \%$ & 49 & $100 \%$ \\
\hline Philosophie & 0 & $0 \%$ & 0 & $0 \%$ & 41 & $100 \%$ \\
\hline SIC & 0 & $0 \%$ & 0 & $0 \%$ & 15 & $100 \%$ \\
\hline Divers & 3 & $4 \%$ & 4 & $6 \%$ & 69 & $100 \%$ \\
\hline Total & 24 & $2 \%$ & 62 & $6 \%$ & 1046 & $100 \%$ \\
\hline
\end{tabular}

Représentativité des bases par disciplines scientifiques (SHS)

\footnotetext{
${ }^{19}$ Sciences de l'information et de la communication
} 